\title{
AN OPERATOR-VALUED MOMENT PROBLEM
}

\author{
LUMINITA LEMNETE
}

(Communicated by Palle E. T. Jorgensen)

\begin{abstract}
We link Carey's exponential representation of the determining function of a perturbation pair with the moment problem. We prove that an operator sequence represents the moments of a phase operator if and only if there is another positively defined sequence of operators satisfying a boundedness condition.
\end{abstract}

\section{INTRODUCTION}

The $L$-moment problem consists in characterizing the moment sequence

$$
A_{n}=\int_{\mathbb{R}} t^{n} f(t) d t, \quad n \in \mathbb{N},
$$

of a measurable function $f$ (with prescribed support in $\mathbb{R}$ ) which satisfies $0 \leq f \leq L$ a.e. This problem was formulated and completely solved by Achiezer and Krein in the 1930s [1]. The problem may be formulated for operator-valued functions. On the other hand, R. W. Carey introduced in [2] a complete unitary invariant which occurs in the perturbation theory of self-adjoint operators. This invariant, called in [2] the "phase shift," is a direct generalization of the phase shift which has been encountered in perturbation situations of one-dimensional range.

Carey proved in [2] that, for $z$ a complex number with $\operatorname{Im} z \neq 0$ and $R_{z}=(A-z)^{-1}$, the determining function

$$
\phi(z)=I+K R_{z} K^{*}
$$

can be represented in the form:

$$
\phi(z)=\exp \left(\int_{\mathbb{R}} B(\lambda) /(\lambda-z) d \lambda\right),
$$

where $B$ is a summable operator function $B: \mathbb{R} \rightarrow \mathscr{L}(\mathscr{X})$ with $0 \leq B(\lambda) \leq$ 1. In the same paper he also proved the converse result: suppose $B(\lambda)$ is a

Received by the editors February 8, 1990 and, in revised form, June 29, 1990; presented at the International Colloquium on complex analysis and the VIth Romanian-Finnish Seminar, Bucharest, June 5-8, 1989.

1980 Mathematics Subject Classification (1985 Revision). Primary 44A60; Secondary 47A55.

Key words and phrases. L-problem of moments, phase operator, determining function. 
summable function with values in $\mathscr{B}(\mathscr{X}, \mathscr{X})$ such that $0 \leq B(\lambda) \leq 1$; then

$$
\phi(z)=\exp \left(\int_{\mathbb{R}} B(\lambda) /(\lambda-z) d \lambda\right)
$$

is the determining function of a certain perturbation pair $\{A, K\}$ with $A$ a selfadjoint operator acting on the Hilbert space $\mathscr{H}$, and $K: \mathscr{H} \rightarrow \mathscr{X}$ with $\mathscr{X}$ another Hilbert space. The function $B(\lambda)$ is called in [2] the phase operator corresponding to the perturbation pair $\{A, K\} . B(\lambda)$ is a complete unitary invariant associated with the perturbation problem $A \rightarrow A+K^{*} K$.

This paper studies the relationship between the two previously mentioned concepts. Hence we prove that the operator sequence $\left(A_{n}\right)_{n=0}^{\infty}$ represents the moments of a summable function

$$
B: \mathbf{R} \rightarrow \mathscr{L}(\mathscr{H}), \quad 0 \leq B(t) \leq 1,
$$

with $\operatorname{supp} B$ compact if and only if we can find another sequence of operators $\left(A_{m}^{\prime}\right)_{m=0}^{\infty}$ positively defined (see the definition given in the next section), with a certain boundedness condition and for which we have the relations

$$
\exp \left(-\sum_{n=0}^{\infty} A_{n} z^{-n-1}\right)=I-\sum_{m=0}^{\infty} A_{m}^{\prime} z^{-m-1} .
$$

The main ingredients of this proof are the two results contained in [2].

\section{THE MAIN RESULT}

Our aim is to prove the following:

Theorem. The sequence $\left(A_{n}\right)_{n=0}^{\infty}$ represents the successive moments of a summable operator function $B(\lambda), 0 \leq B(\lambda) \leq 1$, with $\operatorname{supp} B$ compact if and only if there is an operator sequence $\left(\bar{A}_{m}^{\prime}\right)_{m=0}^{\infty}$ such that

$$
\exp \left(-\sum_{n=0}^{\infty} A_{n} z^{-n-1}\right)=I-\sum_{m=0}^{\infty} A_{m}^{\prime} z^{-m-1},
$$

with both sequences $\left(A_{m}^{\prime}\right)_{m=0}^{\infty}$ and $\left(-A_{m+k+2}^{\prime}+C A_{m+k}^{\prime}\right)_{m, k}$ positively defined for $C$ a positive constant.

Remark. The condition of positive definition for $\left(-A_{m+k+2}^{\prime}+C A_{m+k}^{\prime}\right)_{m, k}$ can be reformulated as a boundedness condition.

Proof. We assume first that $\left(A_{n}\right)_{n=0}^{\infty}$ represents the sequence of successive moments of a summable operator function $B: \mathbf{R} \rightarrow \mathscr{L}(\mathscr{H}), 0 \leq B(\lambda) \leq 1$, i.e.,

$$
A_{n}=\int_{\mathbb{R}} t^{n} B(t) d t=\int_{\operatorname{supp} B} t^{n} B(t) d t .
$$

The first step is a reduction of the power series of the moments (1.1) to a Cauchy integral formula $(*)$. 
Computing the sum for $\mathbf{N}$-indices, we obtain

$$
\begin{aligned}
\sum_{n=0}^{\infty} A_{n} z^{-n-1} & =\sum_{n=0}^{\infty} z^{-n-1} \int_{\mathbb{R}} t^{n} B(t) d t \\
& =\sum_{n=0}^{\infty} \int_{\mathbb{R}} 1 / z(t / z)^{n} B(t) d t
\end{aligned}
$$

for $|z|>|t|$ outside the supp of $B$. From this we obtain

$$
-\sum_{n=0}^{\infty} \int_{\mathbb{R}} 1 / z(t / z)^{n} B(t) d t=-\int_{\mathbb{R}}(B(t) /(z-t)) d t .
$$

Next we build the sequence $\left(A_{m}^{\prime}\right)_{m=0}^{\infty}$ using Carey's result on the phase shift [2]:

If $B$ is a $\mathscr{B}(\mathscr{X}, \mathscr{X})$-valued operator function, so that $0 \leq$ $B(\lambda) \leq 1$, then

$$
\phi(z)=\exp \left(\int_{\mathbb{R}}(B(t) /(t-z)) d t\right)
$$

is the determining function of the perturbation pair $(A, K)$ on $\{\mathscr{H}, \mathscr{K}\}$, where $A$ is a selfadjoint operator acting on the Hilbert space $\mathscr{H}$, and $K$ an operator from $\mathscr{H}$ into another Hilbert space $\mathscr{X}$, i.e.,

$$
\phi(z)=1+K R_{z} K^{*} \text { with } R_{z}=(A-z)^{-1} .
$$

From the above theorem it follows that

$$
\begin{aligned}
K R_{z} K^{*} & =K(A-z)^{-1} K^{*}=-K z^{-1}\left(\sum_{n=0}^{\infty}(A / z)^{n}\right) K^{*} \\
& =-K\left(\sum_{n=0}^{\infty} A^{n} z^{-n-1}\right) K^{*}=-\sum_{n=0}^{\infty} z^{-n-1} K A^{n} K^{*}
\end{aligned}
$$

We identify $A_{m}^{\prime}=K A^{m} K^{*}$; then $\phi(z)$ becomes

$$
\phi(z)=I+K R_{z} K^{*}=I-\sum_{n=0}^{\infty} z^{-n-1} K A^{n} K^{*}=I-\sum_{m=0}^{\infty} A_{m}^{\prime} z^{-m-1}
$$

Applying the preceding result to the function $B(\cdot)$ obtained from the sequence of moments in (1.1), we get the required equality:

$$
\exp \left(-\sum_{n=0}^{\infty} A_{n} z^{-n-1}\right)=I-\sum_{m=0}^{\infty} A_{m}^{\prime} z^{-m-1}
$$

We shall prove that the obtained Hankel quadratic form $\sum_{m, k} A_{m+k}^{\prime} x_{m} x_{k}$ is positively defined (i.e., $\sum_{m, k} A_{m+k}^{\prime} x_{m} x_{k} \geq 0$ for every $\left(x_{m}\right) \subset \mathscr{H}$ with finite 
support) and satisfies the next boundedness condition. More precisely, Positivity

$$
\sum_{k, m}\left\langle A_{k, m}^{\prime} x_{k}, x_{m}\right\rangle \geq 0 \quad \forall\left(x_{k}\right) \subset \mathscr{H},
$$

a family with finite support. Indeed,

$$
\begin{aligned}
\sum_{k, m}\left\langle K^{*} A^{m+k} K x_{k}, x_{m}\right\rangle & =\sum_{k, m}\left\langle A^{k} K x_{k}, A^{m} K x_{m}\right\rangle \\
& =\left\langle\sum_{k=0}^{\infty} A^{k} K x_{k}, \sum_{m=0}^{\infty} A^{m} K x_{m}\right\rangle=\left\|\sum_{m=0}^{\infty} A^{m} K x_{m}\right\|>0 .
\end{aligned}
$$

Boundedness condition. We shall prove that there exists a constant $C>0$ so that

$$
\sum_{m, n=0}^{\infty}\left\langle K A^{m+n+2} K^{*} x_{n}, x_{m}\right\rangle \leq \sum_{m, n=0}^{\infty} C\left\langle K A^{m+n} K^{*} x_{n}, x_{m}\right\rangle .
$$

Indeed,

$$
\left\|A \sum_{n=0}^{\infty} A^{n} K^{*} x_{n}\right\| \leq C\left\|\sum_{n=0}^{\infty} A^{n} K^{*} x_{n}\right\|,
$$

which is true for $\sqrt{C}=\|A\|>0$.

Conversely, assume that we have the representation formula

$$
\exp \left(-\sum_{n=0}^{\infty} A_{n} z^{-n-1}\right)=I-\sum_{m=0}^{\infty} A_{m}^{\prime} z^{-m-1}
$$

with $\left(A_{m}^{\prime}\right)_{m=0}^{\infty}$ positively defined and satisfying the boundedness conditions above. Then we shall prove the existence of a summable operator-valued function $B: \mathbb{R} \rightarrow \mathscr{L}(\mathscr{X})$, with $0 \leq B(\lambda) \leq 1 \operatorname{supp} B$ compact, the function that will furnish the moments of the prescribed $\left(A_{n}\right)_{n=0}^{\infty}$ operator sequence, i.e.,

$$
A_{n}=\int_{\mathbb{R}} B(t) t^{n} d t
$$

We shall consider the operator sequence $\left(A_{m}^{\prime}\right)_{m=0}^{\infty}$, to be doubly indexed. With this assumption, $\left(A_{m}^{\prime}\right)_{m=0}^{\infty}$ can be represented as an operator-valued, positively defined function

$$
A^{\prime}: \mathbb{N} \times \mathbb{N} \rightarrow \mathscr{L}(\mathscr{H}), \quad A^{\prime}(m, n)=A_{m+n}^{\prime} .
$$

The classical Kolmogorov theorem gives a decomposition for positively defined kernels:

Let $K: I \times I \rightarrow \mathscr{L}(\mathscr{H})$ be a positively defined operator-valued function (i.e., $\sum_{i, j}\left\langle K(i, j) x_{i}, x_{j}\right\rangle \geq 0$ for every family $\left(x_{i}\right)_{i}$ with finite support). Then $K(i, j)$ admits a decomposition of the form $K(i, j)=h_{i}^{*} h_{j}$, with $h_{i} \in \mathscr{L}(\mathscr{H})$. 
Thus $A_{m+n}^{\prime}$ can be represented as $A_{m+n}^{\prime}=K_{n}^{*} K_{m}$.

From the boundedness and positivity conditions we can find a constant $C>$ 0 such that $\left(-A_{m+k+2}^{\prime}+C A_{m+k}^{\prime}\right)$ is positively defined. From the Kolmogorov decomposition, it follows that $\left(-K_{m+1}^{*} K_{n+1}+C K_{m}^{*} K_{n}\right)$ is positively defined. According to this, for $\left(x_{k}\right)_{k}$ an arbitrary family of vectors of finite support we have

$$
C \sum_{k, m}\left\langle K_{m}^{*} K_{k} x_{k}, x_{m}\right\rangle \geq \sum_{k, m}\left\langle K_{m+1}^{*} K_{k+1} x_{k}, x_{m}\right\rangle,
$$

an inequality which becomes:

$$
\sqrt{C}\left\|\sum_{k=0}^{\infty} K_{k} x_{k}\right\| \geq\left\|\sum_{k=0}^{\infty} K_{k+1} x_{k}\right\| .
$$

We take by definition

$$
A\left(\sum_{k=0}^{\infty} K_{k} x_{k}\right):=\sum_{k=0}^{\infty} K_{k+1} x_{k} .
$$

Since the $K_{n}$ are linear, so is $A$ and, from our previous remarks, $A$ is continuous. Taking $x_{0}=(1,0, \ldots), x_{i}=0, i>1$, we obtain $A K_{0}=K_{1}$, and using the induction method for a suitable choice of $\left(x_{n}\right)_{n=0}^{\infty}$, we obtain $K_{n}=A^{n} K_{0}$. We prove now that $A$ is a selfadjoint operator: For $x$ in a dense subset of $\mathscr{H}$, we can find $\left(x_{k}\right)_{k}$ such that $x=\sum_{k=0}^{\infty} K_{k} x_{k}$. In this case,

$$
\langle A x, x\rangle=\left\langle\sum_{k=0}^{\infty} K_{k+1} x_{k}, \sum_{k=0}^{\infty} K_{k} x_{k}\right\rangle \in \mathbb{R},
$$

because $K_{k}^{*} K_{k+1}$ are positively defined. Thus, $\langle A x, x\rangle \in \mathbb{R}$, and so $A$ is a selfadjoint operator.

With this assumption, (1.2) will be rewritten in the form

$$
\begin{aligned}
\exp \left(-\sum_{n=0}^{\infty} A_{n} z^{-n-1}\right) & =I-\sum_{n=0}^{\infty} K_{0}^{*} A^{n} K_{0} z^{-n-1} \\
& =I-\sum_{n=0}^{\infty} 1 / z K_{0}^{*}(A / z)^{n} K_{0}=I+K_{0}^{*} R_{z} K_{0}=\phi(z),
\end{aligned}
$$

where $R_{z}=(A-z)^{-1}$ and $A=A^{*}, K=K_{0}^{*}$.

From this calculation we obtain the determining function of the perturbation pair $\{A, K\}$ :

$$
\phi(z)=I+K_{0} R_{z} K_{0}^{*} \quad \text { and } \exp \left(-\sum_{n=0}^{\infty} A_{n} z^{-n-1}\right)=\phi(z) .
$$

The perturbation function is a holomorphic operator-valued function satisfying

(1.1) $\phi(z)^{*}=\phi(\bar{z})$.

(1.2) $1 / 2 i\left\{\phi(z)-\phi(z)^{*}\right\}=\operatorname{Im} \phi(z) \geq 0$ for $\operatorname{Im} z>0$.

(1.3) $\|\phi(z)-1\|=O(1 / \operatorname{Im} z)$ as $|\operatorname{Im} z| \rightarrow \infty$. 
We are now in the situation of applying the main theorem from [2]:

Suppose $\phi(z)$ is the determining function of a perturbation pair $\{A, K\}$ on the Hilbert spaces, $A$ a selfadjoint operator acting on $\mathscr{H}$, and $K: \mathscr{H} \rightarrow \mathscr{X}$. There exists a summable function $B(\lambda)$ with values in the set of positive operators of the unit ball of $\mathscr{B}(\mathscr{X}, \mathscr{X})$ such that

$$
\phi(z)=\exp \left(\int_{\mathbb{R}}(B(\lambda) /(\lambda-z)) d \lambda\right), \quad \operatorname{Im} z \neq 0 .
$$

Representing $\phi(z)$ in power series, we have

$$
\begin{aligned}
\phi(z) & =\exp \left(-\int_{\mathbb{R}}(B(\lambda) / z(1-\lambda / z)) d \lambda\right) \\
& =\exp \left(-\sum_{n=0}^{\infty} z^{-n-1} \int_{\mathbb{R}} B(\lambda) \lambda^{n} d \lambda\right) .
\end{aligned}
$$

From both representations (1.3) and (1.4),

$$
A_{n}=\int_{\mathbb{R}} B(\lambda) \lambda^{n} d \lambda
$$

which ends the proof of the main theorem.

\section{FINAL REMARKS}

The support of function $B$ can be characterized in terms of its moments, as in the scalar case (cf. [1]). Our approach also gives a necessary condition for the $L$-problem of moments with operator values, without restriction on the support.

\section{ACKNOWLEDGMENT}

The author would like to thank Mihai Putinar for his useful advice.

\section{REFERENCES}

1. N. I. Achiezer and M. G. Krein, Some questions in the theory of moments, Amer. Math. Soc., Providence, RI, 1962.

2. R. W. Carey, Unitary invariant for self-adjoint operators, 1973.

3. M. Putinar, A L-problem of moments in two-dimensions, preprint, 1988.

4. D. Sarason, Moment problems and operators in Hilbert space, Proc. Sympos. Appl. Math., vol. 37, Amer. Math. Soc., Providence, RI, 1987, p. 54.

5. F. H. Vasilescu, Introducere in teoria operatorilor liniari, Editura Tehnicā, 1987.

Research Institute for Computer Technology and Informatics, Calea Floreasca 167, Sector 2, POSTE CODE 72321, Bucharest, RoMANia

Current address: Department of Mathematics I, Polytechnic Institute of Bucharest, Splaiul Independentei 313, Sect. 6 Poste Code 77206 Bucharest, Romania 